\title{
Evaluation de la Vulnérabilite à l'Inondation des Communes à Proximité des Grandes Villes Ouest Africaines : Cas de la Commune de Bingerville (Est d'Abidjan - Côte d'Ivoire)
}

\author{
Anowa Evrade Larissa Eba \\ Gabriel Etienne Ake \\ D'avila Françoise Gouadou
}

Univertité Félix Houphouët-Boigny, UFR des Sciences de la Terre et des Ressources Minières, Laboratoire des Sciences du Sol, de l'Eau et des Géomatériaux (LSSEG), Abidjan, Côte d'Ivoire

\section{Jeanpatrice Jourda}

Univertité Félix Houphouët-Boigny, UFR des Sciences de la Terre et des Ressources Minières, Laboratoire des Sciences du Sol, de l'Eau et des

Géomatériaux (LSSEG), Abidjan, Côte d'Ivoire

Centre Universitaire de Recherche et d'Application en Télédetection (CURAT), UFR des Sciences de la Terre et des Ressouces Minières,

Abidjan, Côte d'Ivoire

\section{Doi:10.19044/esj.2021.v17n14p277}

Submitted: 14 January 2021

Accepted: 10 March 2021

Published: 30 April 2021
Copyright 2021 Author(s)

Under Creative Commons BY-NC-ND

4.0 OPEN ACCESS

Cite As:

Eba A.E.L,, Ake G.E,, Gouadou D.F. \& Jourda J. (2021). Evaluation de la Vulnérabilite à l'Inondation des Communes à Proximité des Grandes Villes Ouest Africaines : Cas de la Commune de Bingerville (Est d'Abidjan - Côte d'Ivoire). European Scientific Journal, ESJ, 17(14), 277. https://doi.org/10.19044/esj.2021.v17n14p277

\section{Resume}

Cette étude vise à mettre en évidence des zones potentiellement inondables ou à risque d'inondation dans la commune de Bingerville (à l'Est du district d'Abidjan) dans un contexte d'urbanisation rapide et non maitrisée, en vue d'une gestion intégrée et durable de son espace. La démarche méthodologique utilisée a consisté à combiner les techniques de l'analyse multicritère, et les systèmes d'informations géographiques. Cinq paramètres que sont: la pente, l'occupation du sol, la densité de drainage, la densité de population et les facteurs environnementaux enthropiques ont été utilisés dans cette étude. Ensuite, des poids ont été assignés à ces paramètres par 
l'application de la méthode d'analyse multicritère de Saaty (AHP). La carte de vulnérabilité, a été obtenue par la combinaison des cinq paramètres à travers un SIG, et elle révèle cinq classes (très faible, faible, moyenne, fort et très forte). Les résultats montrent que la commune de Bingerville est moyennement vulnérable à l'inondation. Cependant, certains quartiers situés principalement au centre de la commune et en zones périphériques et certains villages situés en bordure de lagune tels que Anan et Akandjè présentent de fortes vulnérabilités à l'inondation. Cette information scientifique oriente des prises de décisions par les autorités en charge de la gestion de la commune dans la prévention et la prise en charge des crises d'inondation.

Mots clés : Evaluation, Vulnérabilité, Inondation, Bingerville, Abidjan

\title{
Flood Vulnerability Assessment of Communes in the Vicinity of Large West African Cities: Case of Bingerville Commune (East of Abidjan - Côte d'Ivoire)
}

\section{Anowa Evrade Larissa Eba \\ Gabriel Etienne Ake \\ D'avila Françoise Gouadou}

Univertité Félix Houphouët-Boigny, UFR des Sciences de la Terre et des Ressources Minières, Laboratoire des Sciences du Sol, de l'Eau et des

Géomatériaux (LSSEG), Abidjan, Côte d'Ivoire

\section{Jeanpatrice Jourda}

Univertité Félix Houphouët-Boigny, UFR des Sciences de la Terre et des Ressources Minières, Laboratoire des Sciences du Sol, de l'Eau et des Géomatériaux (LSSEG), Abidjan, Côte d'Ivoire

Centre Universitaire de Recherche et d'Application en Télédetection (CURAT), UFR des Sciences de la Terre et des Ressouces Minières, Abidjan, Côte d'Ivoire

\begin{abstract}
This study aims to highlight potentially floodable areas or areas at risk of flooding in the town of Bingerville (East of the district of Abidjan) in a context of rapid and uncontrolled urbanization, for an integrated and sustainable management of its space. The methodological approach used consisted in combining the techniques of multicriteria analysis, and geographic information systems. Five parameters including slope, land use, drainage density, population density and anthropogenic factors were used in this study. Then, weights were attributed to these parameters through Saaty's
\end{abstract}


multi-criteria method (AHP). The vulnerability to flood was categorized into five classes (very low, low, medium, high and very high) by using GIS. The results show that the commune of Bingerville is moderately vulnerable to flooding. However, some neighborhoods located mainly in the center of the commune and in peripheral areas and some villages located on the edge of the lagoon such as Anan and Akandjé are highly vulnerable to flooding. This scientific information is used to guide decision-making by the authorities in charge of managing the commune in the prevention and management of flood crises.

Keywords: Assessment, Vulnerability, Flood, Bingerville, Abidjan

\section{Introduction}

Ces dernières années, à l'instar des autres continents, de violentes inondations ont été signalées dans de nombreux pays africains. C'est le cas du Burkina Faso, du Mali, de la Mauritanie, du Niger et du Sénégal qui, en 2003 ont été touchés par des inondations causant la mort de plusieurs dizaines de personnes et la perte des récoltes (Nouaceur, 2015).

La Côte d'Ivoire ne reste pas en marge de ces catastrophes. En effet, ses villes ont été affectées par des inondations d'origines diverses ces deux dernières décennies. En 2018, les fortes pluies ont causé la mort de 20 personnes dans le pays et couté près de 18 milliards de pertes et de dommages (Jeune Afrique, 2020). De la période de 2009 à 2014, il a été dénombré au total 74 décès occasionnés par les inondations dans le District d'Abidjan (Koffi et al., 2014). Egalement, selon le bilan du plan National en 2020, à la date du 21 juin, dans la seul ville d'Abidjan, on enregistrait près de 721 ménages sinistrés, soit 3605 personnes affectées, 9 blessées et 19 décès. Selon Chérif (2014), ces catastrophes seraient causées par des phénomènes naturels liés aux changements climatiques dont la montée des eaux de mer comme le cas du quartier de Gonzagueville au Sud de la ville d'Abidjan. Parallèlement aux effets naturels, la population à travers les constructions anarchiques dans les bassins d'orage et sur le réseau d'assainissement contribue à la mise en place des zones inondables à travers le comblement progressif des ouvrages de collecte des eaux à ciel ouvert, par des dépôts solides de toutes sortes, empêchant ainsi le drainage des eaux pluviales (Koffi et al., 2014). De même, l'urbanisation rapide de la ville d'Abidjan, avec son corolaire de problèmes de logement et de nuisances sonores, motive les populations à s'orienter vers les nouvelles communes huppées en périphérie comme Bingerville qui est en plein essor. Ainsi, d'après l'INS (2014), le taux d'accroissement annuel moyen enregistré à Bingerville entre 1998 et 2014, est de 3,2\%. Malheureusement, selon les agents du service technique de la mairie de Bingerville, certains promoteurs immobiliers, ou particuliers, construisent 
dans les ravins, cuvettes sans aménagement préalable et sans tenir compte du plan de construction des villes préétabli par l'Etat de Côte d'Ivoire. Fort de ce constat, la présente étude a été entreprise dans l'optique de mettre en évidence les zones potentiellement vulnérables à l'inondation dans la commune de Bingerville afin de faciliter la prise de décision pour la sécurité des biens et des populations.

\section{Cadre General de la Zone d'etude}

D'une superficie de $10200 \mathrm{~km}^{2}$, Bingerville est une commune du Sud de la Côte d'Ivoire située précisément à l'Est de la ville d'Abidjan, entre les latitudes $5^{\circ} 16^{\prime}$ et $5^{\circ} 25^{\prime} \mathrm{N}$ et les longitudes $3^{\circ} 43^{\prime}$ et $3^{\circ} 55^{\prime} \mathrm{W}$. Elle est limitée au Sud par les communes de Port-Bouët et de Grand-Bassam, à l'Est par la préfecture d'Alépé, au Nord par la commune d'Anyama et à l'Ouest par la commune de Cocody. Ses limites naturelles sont la lagune Ebrié au Sud, et les lagunes Aghien et Potou respectivement au Nord-ouest et au Nord-est (Figure $1)$.

Sur le plan démographique, la population de Bingerville est estimée à 91319 habitants, selon le Recensement Général de la Population et de l'Habitat (RGPH, 2014). La commune de Bingerville se trouve dans le bassin sédimentaire émergé constitué par un manteau plus ou moins épais de sables, d'argiles, de sables argileux et de grès ferrugineux qui recouvrent les dépôts anciens (Assalé et al., 2012). Elle est traversée par un vaste système lagunaire constitué des lagunes Ebrié, Aghien et Potou.

La pluviométrie moyenne annuelle est de l'ordre de $1720,7 \mathrm{~mm}$ sur la période 1960 à 2014 selon la Société d'Exploitation et de Développement Aéroportuaire, Aéronautique et Météorologique (SODEXAM), avec une évapotranspiration réelle de $979,19 \mathrm{~mm}$, soit $56,91 \%$ de la lame d'eau tombée. Environ 43,09\% des pluies tombées ruissellent vers les lagunes. Aussi, le regain pluviométrique observé cette dernière décennie associé à l'urbanisation rapide et non réglementée sont à l'origine de ce fort ruissellement urbain, provoquant des inondations pendant les mois pluvieux de mai et juin (Tah et al., 2018). 


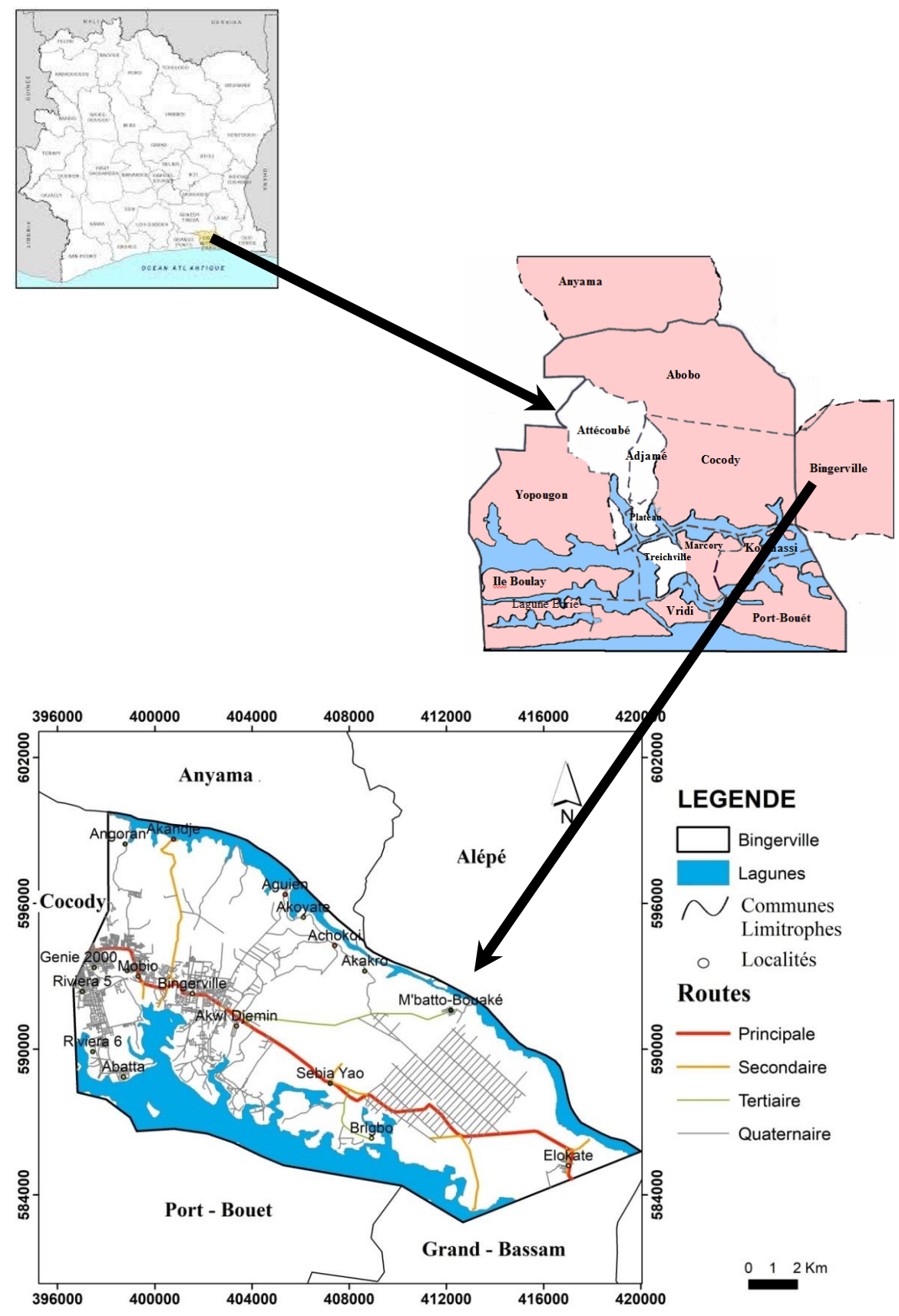

Figure 1: Localisation de la zone d'étude

\section{Materiel et Methode}

\section{II.1 Données}

La mise en place d'une base de données a été nécessaire pour mener à bien cette étude. Ces données utilisées sont : 
- une image satellitaire Landsat OLI 8 de résolution 30m, datant du 28 décembre 2018, et téléchargée à l'adresse : https://earthexplorer.usgs.gov. Elle a permis d'établir la carte d'occupation du sol ;

- le Model Numérique de Terrain (MNT) de résolution 30m couvrant la Côte d'Ivoire. Elle a permis d'établir la carte des pentes et de densité de drainage de la zone d'étude ;

- les résultats du recensement des populations de 1998 et 2014 de la commune de Bingerville qui a permis d'établir la carte de densité de la population.

Les logiciciels utilisés pour le traitement de ces données sonts ENVI 5.1 pour le traitement des images satellitaires et ArcGIS 10.4 pour l'établissement des différentes cartes.

\section{II.2 Approche Méthodologique}

L'approche méthodologique utilisée dans cette étude est basée sur les investigations socio-environnementales dominées par des observations de terrains, la cartographie, l'analyse multicritère et les SIG.

\section{II.2.1 Investigation socio-environnementale}

Elle a été effectuée dans le mois de mai 2019, avant les fortes pluies. Elle a consisté à identifier les différents quartiers de la commune de Bingerville et observer les zones les plus susceptibles d'être inondées en se basant sur leur topographie (zone de bas-fonds).

\section{II.2.2 Spatialisation des zones à risque d'inondation et potentiellement inondables}

La méthodologie utilisée pour cartographier la vulnérabilité à l'inondation dans la commune de Bingerville est basée sur la méthode d'analyse hiérarchique multicritère (AHP) développée par Saaty (1984). Plusieurs auteurs dont: Danumah, (2013), Yeo (2017), Balliet (2017) l'ont utilisé pour l'analyse des risques d'inondation. D'après Mulders (2001) l'utilisation d'une approche multicritère permet la prédiction spatiale des mouvements superficiels (glissements de terrain, fluages, inondations etc.) et l'application d'analyses multiples aboutit à des cartographies thématiques fiables.

Cette méthodologie comporte cinq étapes à savoir :

- identification et hiérarchisation des critères ;

- classification et standardisation des critères ;

- pondération des critères ; 
- calcul de l'indice de cohérence IC ;

- agrégation des critères à travers un SIG.

\section{II.2.2.1. Identification des critères naturels et anthropiques}

Pour des raisons de disponibilité des données et de l'état d'urbanisation intense de la zone d'étude, les critères ou facteurs suivants sont utilisés pour la cartographie de la vulnérabilité à l'inondation de la zone d'étude. Il s'agit de : l'occupation du sol, la pente topographique, la densité de drainage, la densité de population et les facteurs environnementaux anthropiques.

- Occupation du sol (OS)

L'occupation du sol joue un rôle prépondérant dans le ruissellement des eaux. Le système racinaire améliore la perméabilité du sol, le feuillage protège le sol de l'impact de la pluie et diminue le phénomène de battance (Musy et Soutter, 1991 ; Haouchine et al., 2010). Par ailleurs, le couvert végétal permet aussi le confinement de l'eau en-dessous de sa zone de couverture, diminuant ainsi le taux d'évaporation directe. Une terre nue ou imperméabilisée aura tendance à faire augmenter le ruissellement et l'érosion (Douay et Lardieg, 2010).

La figure 2 ci-dessous présente les différentes occupations du sol de la zone d'étude.

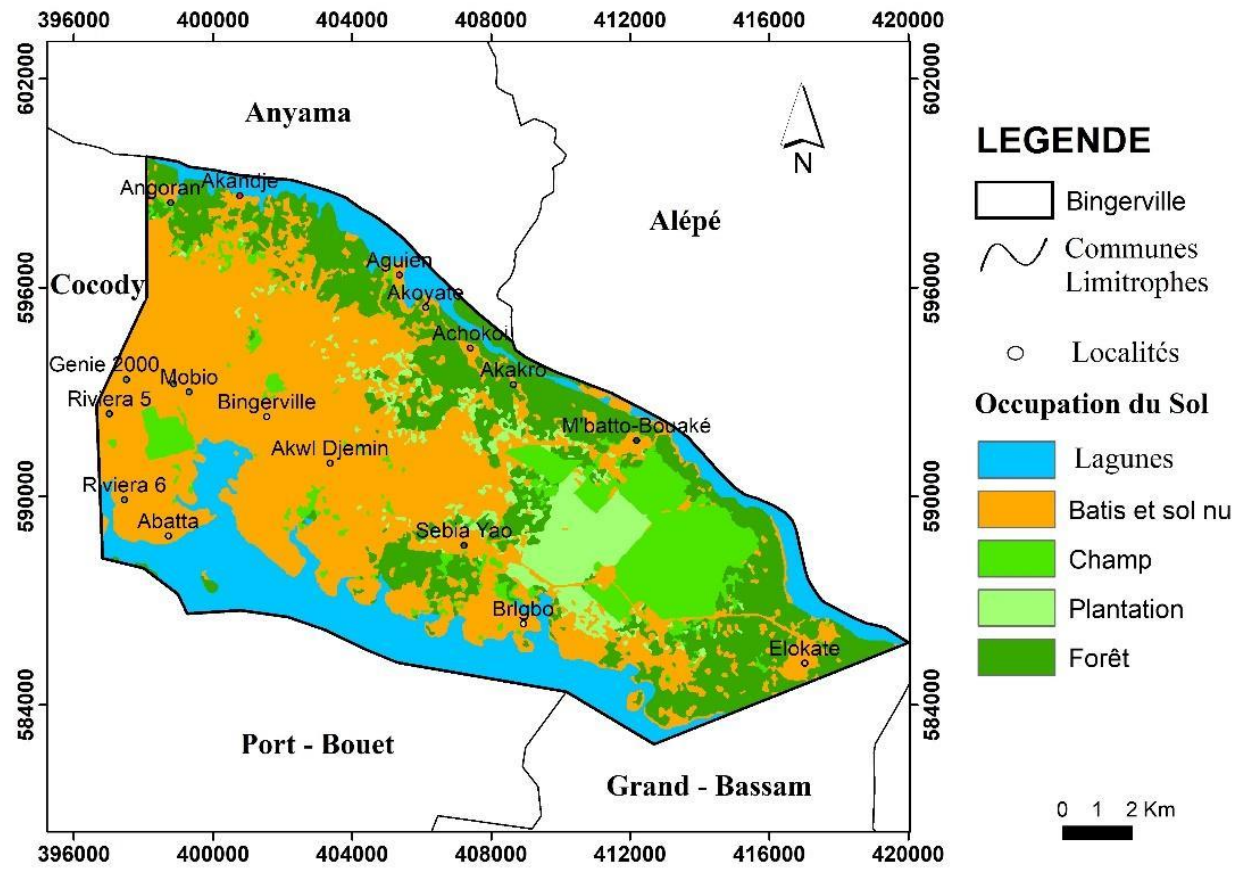

Figure 2: Carte de l'occupation du sol de la commune de Bingerville 
- Pente $(\mathrm{P})$

La pente est une caractéristique géomorphologique qui a une influence sur l'écoulement superficiel de l'eau dans une zone donnée. En définitif, elle traduit les zones potentiellement favorables au ruissellement ou à l'infiltration avec le réseau hydrographique pris comme représentatif de la réalité de la répartition du ruissellement (Koffi, 2016).

La carte des pentes est générée à partir du modèle numérique de terrain (MNT) de la zone d'étude extrait à partir de celle de la Côte d'Ivoire. Puis l'utilisation de l'outil "Slope" dans le logiciel ArcGIS permet de mettre en évidence les pentes qui sont par la suite classées grâce à l'outil "reclassify".

La figure 3 présente les différentes pentes obtenues dans la zone d'étude.

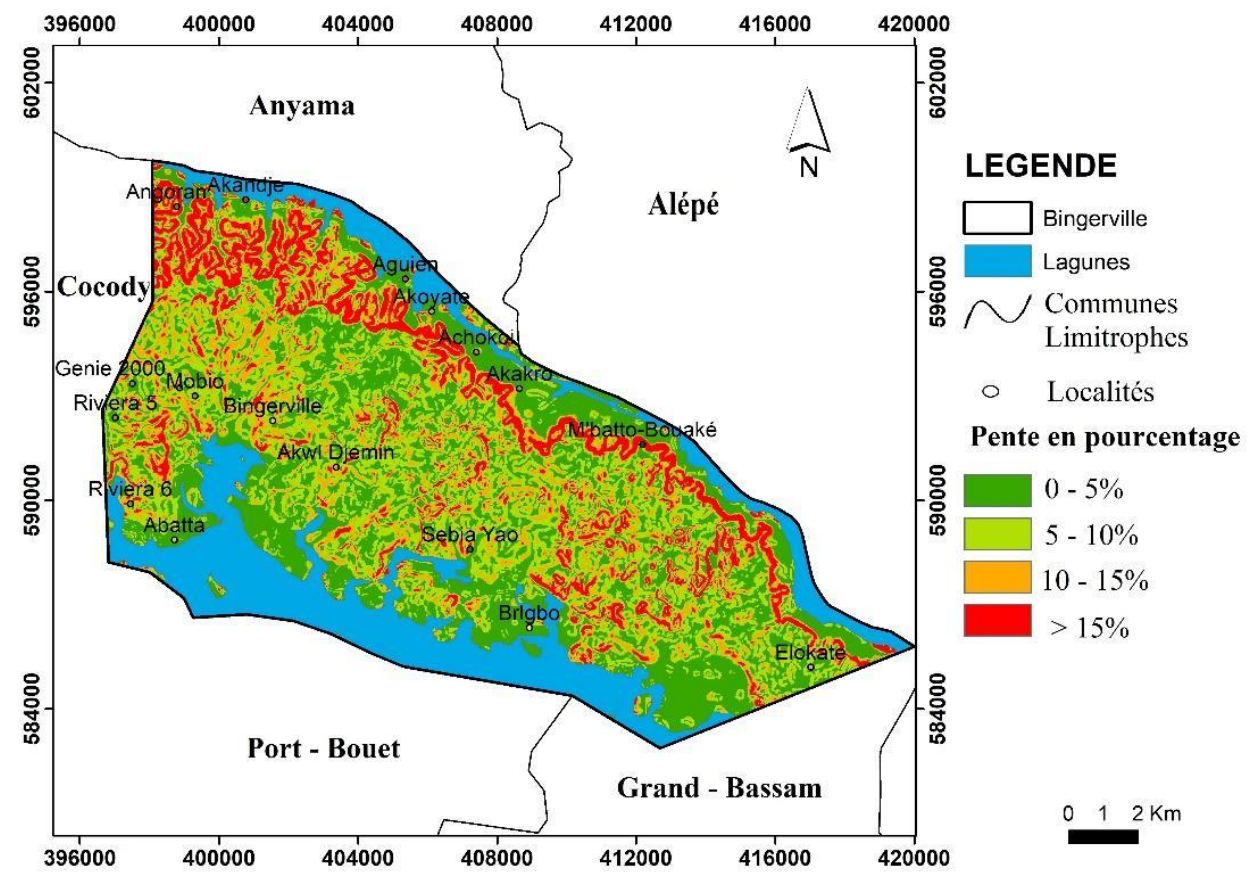

Figure 3 : Carte des pentes de la commune de Bingerville

- Densité de drainage (DD)

La densité de drainage dépend de la géologie (structure et lithologie) et des caractéristiques topographiques de la zone étudiée. Elle est liée à la densité de réseau hydrographique.

En effet, plus le réseau hydrographique est dense, plus le phénomène de ruissellement est intense donc l'infiltration est faible (Koffi, 2016). La carte de densité de drainage est réalisée sur la base du réseau hydrographique de la 
zone d'étude sur lequel l'outil "Line density" du logiciel ArcGis est appliqué. La figure 4 permet de mettre en évidence le drainage de la zone.

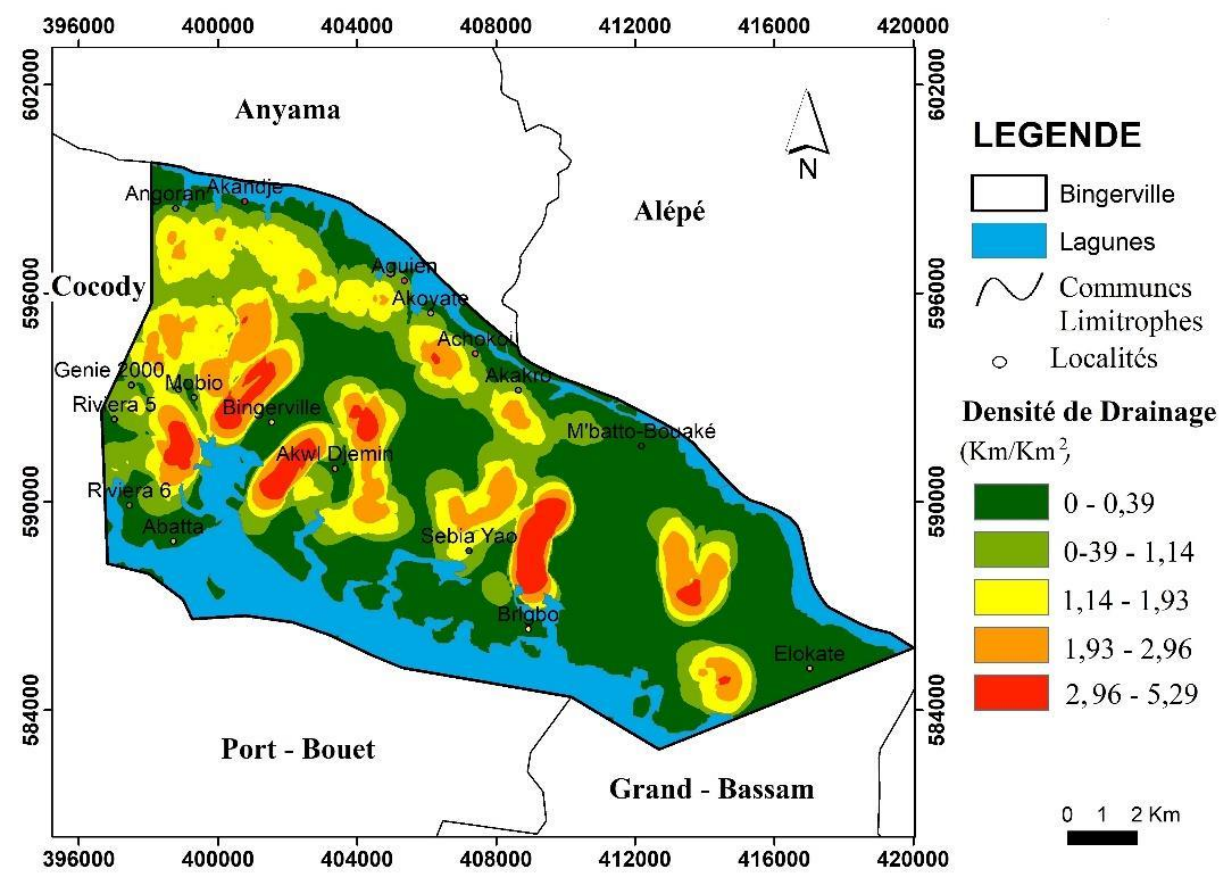

Figure 4: Carte de densité de drainage de la commune de Bingerville

- Densité de population (DP)

La démographie est un facteur qui intervient dans le phénomène d'inondation. En effet, le fait qu'une zone soit fortement peuplée indique une réduction de la végétation, qui fait place aux infrastructures de toutes sortes.

La carte de densité de population est obtenue à partir des données de recensement de la population (RGPH, 2014) dans chaque quartier et village de ladite commune. En effet, ces données ont été introduites dans le logiciel ArcGIS puis interpolées afin de mettre en évidence le nombre d'habitants par $\mathrm{Km}^{2}$ (figure 5). 


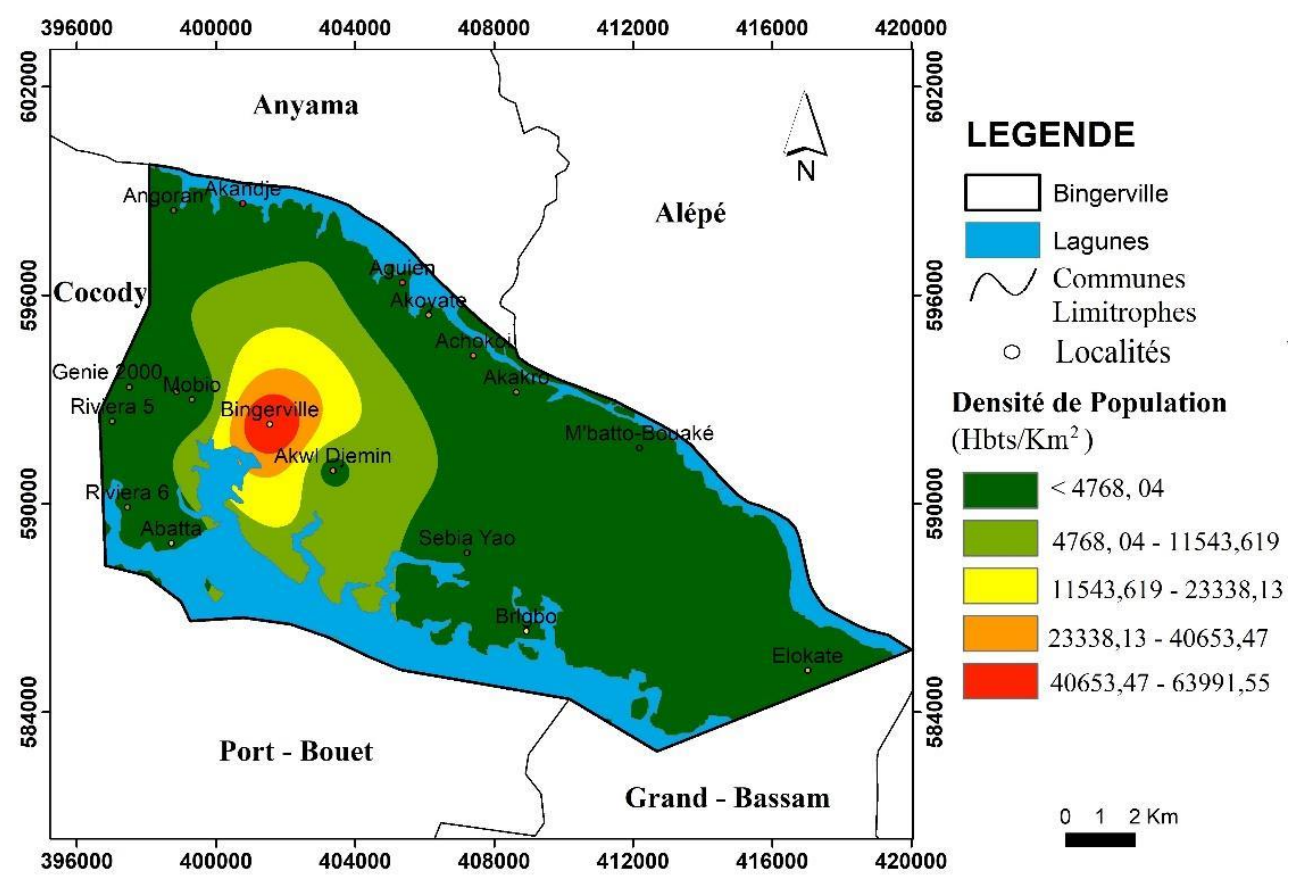

Figure 5: Carte de densité de population de la commune de Bingerville

- Facteurs environnementaux anthropiques (FEA)

L'augmentation de la démographie de la commune de Bingerville (56356 habitants en 1998 à 91319 habitants en 2014) entraîne nécessairement l'intensification de l'urbanisation, d'où l'accentuation des facteurs environnementaux anthropiques intervenant dans le phénomène d'inondation. Ce sont entre autres :

- la dégradation du réseau de drainage ;

- les dépôts sauvages d'ordures ;

- les constructions anarchiques en particulier dans les zones marginales (bas-fonds, cuvettes et marécages).

Le comportement des populations qui favorise l'inondation est leur capacité à s'installer dans les bas-fonds et sur les canalisations d'eaux pluviales. Aussi lorsque les populations utilisent les canalisation d'eaux pluviales pour le drainage des eaux usées domestiques, cela entraine un débordement de celles-ci.

La carte des facteurs environnementaux anthropiques a été obtenue à la suite d'une enquête géographique réalisée pour collecter des données pouvant occasionner les inondations. Elle s'est effectuée du 16 au 26 septembre 2019. 
Elle a consisté à une prise de coordonnées de certains facteurs environnementaux anthropiques choisis (dépôts sauvages d'ordures, réseau de drainage, infrastructures routières), à une description de ceux-ci, ainsi qu'à la rencontre faite avec des agents du service technique de la mairie de la commune.

La figure 6 montre la répartition des différents facteurs anthropiques recensés.

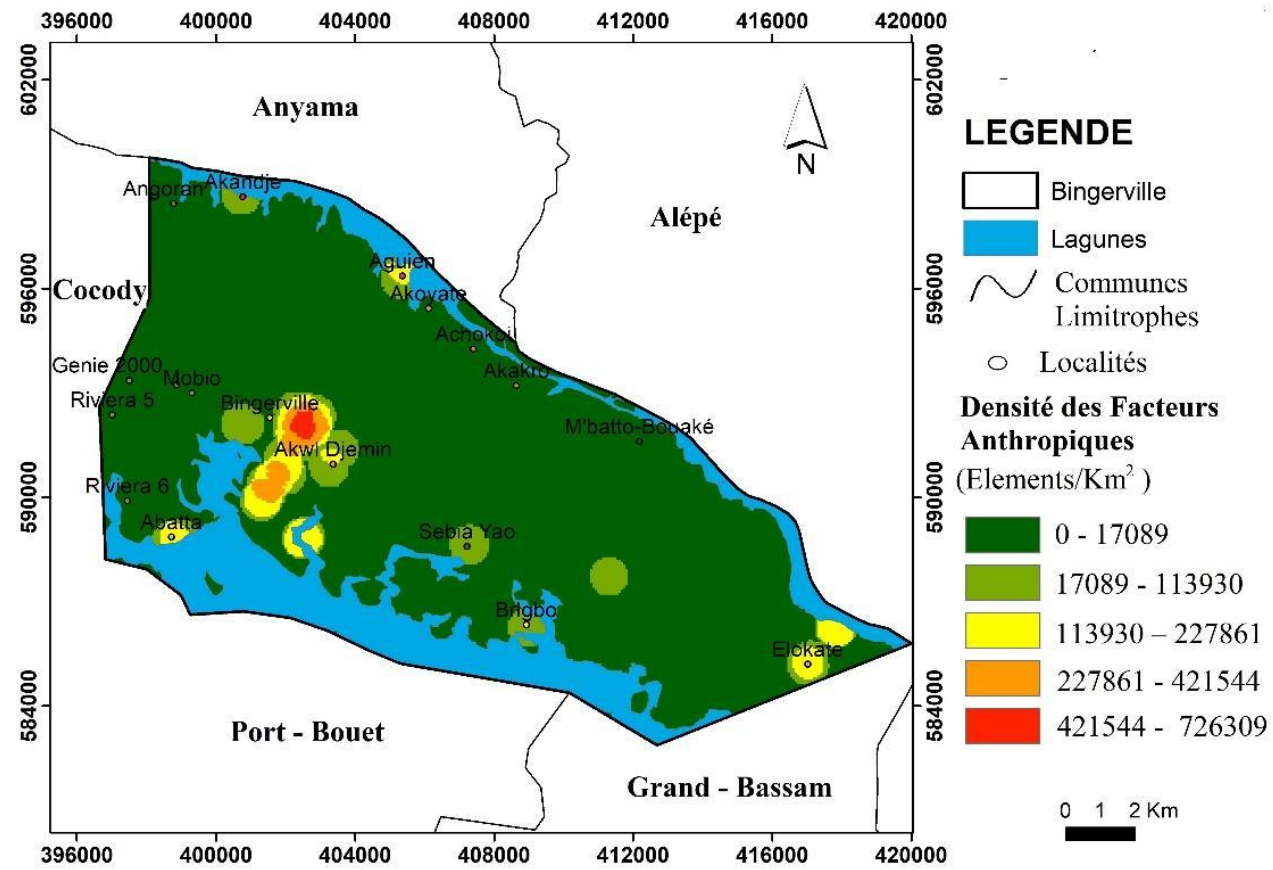

Figure 6: Carte de densité des facteurs anthropiques de la commune de Bingerville

Une fois définis, les facteurs ont été organisés en niveaux homogènes selon le principe de construction hiérarchique.

\section{II.2.2.2 Classification et standardisation des critères identifiés}

Les critères étant mesurés sur des échelles différentes, avec des unités différentes, une standardisation de ceux-ci s'impose pour une bonne analyse multicritère.

En effet, dans le cadre de l'étude de la recharge potentielle des aquifères, une échelle des cotes a été élaborée par Haouchine et al., (2010) afin d'estimer les cotes des différents critères de façon homogène, sur la base des plages de variation des critères proposés (Tableau I). 
Tableau 1: Echelle d'évaluation des différents facteurs (Haouchine et al., 2010)

\begin{tabular}{|c|c|c|c|c|c|c|c|}
\hline Classes & $\begin{array}{l}\text { Très } \\
\text { Forte }\end{array}$ & Forte & $\begin{array}{l}\text { Forte à } \\
\text { Moyenne }\end{array}$ & Moyenne & $\begin{array}{l}\text { Moyenne } \\
\text { à Faible }\end{array}$ & Faible & $\begin{array}{l}\text { Très } \\
\text { Faible }\end{array}$ \\
\hline Cotes & 10 & 8 & 6,5 & 5 & 3,5 & 2 & 1 \\
\hline
\end{tabular}

Pour la présente étude, nous avons adapté cette échelle à la classification intra paramétrique pour l'inondation. Ce qui suppose que les valeurs de cote attribuées aux classes très fortes dans l'évaluation de Haouchine et al.., (2010) seront attribuées aux classes de cote plus faibles dans cette étude et vis-versa. Ainsi, les classes des différents facteurs ou critères sont codifiées en fonction de leur importance à favoriser les inondations ou non. Par exemple, dans le cas de la densité de drainage, les cotes élevées désigneront les zones faiblement drainées et les cotes faibles désigneront celles fortement drainées. En outre, les intervalles de valeurs des classes ont été obtenues pour certains à partir du logiciel utilisé (Pente, Facteurs Environnementaux Anthropiques etc.) et pour d'autres fixées par l'auteur (Occupation du Sol) ( Tableau II).

Tableau II : Récapitulatif des classes, et cotes attribuées aux différents facteurs

\begin{tabular}{|c|c|c|c|}
\hline Facteurs & Classes & $\begin{array}{c}\text { Intensité } \\
\text { du phénomène }\end{array}$ & Cotes \\
\hline \multirow{5}{*}{$\begin{array}{l}\text { Occupation du Sol } \\
\text { (OS) }\end{array}$} & Forêt & Très faible & 1 \\
\hline & Plantations & Faible & 2 \\
\hline & Champs & Moyen & 5 \\
\hline & Bâtis et sol nu & Fort & 8 \\
\hline & Lagunes & Très fort & 10 \\
\hline \multirow{4}{*}{ Pente $(\%)$} & $0-5 \%$ & Faible & 8 \\
\hline & $5-10 \%$ & Moyen & 5 \\
\hline & $10-15 \%$ & Fort & 2 \\
\hline & $>15 \%$ & Très fort & 1 \\
\hline \multirow{5}{*}{$\begin{array}{l}\text { Densité de drainage } \\
\left(\mathrm{Km} / \mathrm{Km}^{2}\right)\end{array}$} & $0-0,39$ & Très faible & 10 \\
\hline & $0,39-1,14$ & Faible & 8 \\
\hline & $1,14-1,93$ & Moyen & 5 \\
\hline & $1,93-2,96$ & Fort & 2 \\
\hline & $2,96-5,29$ & Très fort & 1 \\
\hline \multirow{5}{*}{$\begin{array}{l}\text { Densité de population } \\
\left(\mathrm{Hbts} / \mathrm{Km}^{2}\right)\end{array}$} & $<4768,04$ & Très faible & 1 \\
\hline & $4768,04-11543,619$ & Faible & 2 \\
\hline & $11543,619-23338,13$ & Moyen & 5 \\
\hline & $23338,13-40653,47$ & Fort & 8 \\
\hline & $40653,47-63991,55$ & Très fort & 10 \\
\hline \multirow{5}{*}{$\begin{array}{l}\text { Densité des facteurs } \\
\text { environnementaux } \\
\text { (Elements/Km2) }\end{array}$} & $0-17089$ & Très faible & 1 \\
\hline & $17089-1113930$ & Faible & 2 \\
\hline & $113930-227861$ & Moyen & 5 \\
\hline & $227861-421544$ & Fort & 8 \\
\hline & $421544-726309$ & Très fort & 10 \\
\hline
\end{tabular}




\section{II.2.2.3. Pondération des facteurs par analyse multicritère de Saaty II.2.2.3.1. Réalisation des combinaisons binaires}

La méthode de Saaty (1977), utilisée par plusieurs auteurs dont El Morjani (2002); Eba et al., (2013); Ake et al., (2019) est exploitée dans cette étude. C'est une méthode de comparaison par paire à travers le Processus d'Analyse Hiérarchique (Analytical Hierarchy Process, AHP).

Il s'agit de comparer l'importance relative de tous les facteurs retenus pris deux à deux pour configurer une matrice carrée réciproque. Cette comparaison se fait sur la base d'une échelle numérique de 9 niveaux de comparaison par paire (Saaty, 1977). Lorsque deux paramètres ont la même importance dans le phénomène étudié, l'échelle de Saaty leur donne la valeur de "1". Cependant, si un paramètre est plus important que l'autre, alors il prend une valeur supérieure comprise entre 1 et 10 et l'autre, l'inverse de cette valeur.

Cette méthode permet de produire des coefficients de pondération standardisés dont la somme est égale à "1". La matrice issue de la comparaison par paire des différents facteurs est résumée dans le tableau III.

Tableau III: Matrice issue de la comparaison des facteurs (Matrice originale)

\begin{tabular}{lccccc}
\hline & OS & P & DD & FE & DP \\
\hline OS & 1,00 & 3,00 & 5,00 & 7,00 & 9,00 \\
\hline $\mathbf{P}$ & $1 / 3$ & 1,00 & 3,00 & 5,00 & 7,00 \\
\hline DD & $1 / 5$ & 13 & 1,00 & 3,00 & 5,00 \\
\hline FE & $1 / 7$ & $1 / 5$ & $1 / 3$ & 1,00 & 3,00 \\
\hline DP & $1 / 9$ & $1 / 7$ & $1 / 5$ & $1 / 3$ & 1,00 \\
\hline Total $\left(\sum \mathbf{a}_{\mathbf{i}}\right)$ & 1,78 & 4,67 & 9,53 & 16,33 & 25 \\
\hline
\end{tabular}

Les comparaisons par binaire sont alors analysées pour calculer les coefficients de pondération $(\mathrm{Cp})$ ou"poids" des différents critères.

Aussi, une vérification de la cohérence des jugements (cohérence logique) estelle effectuée.

\section{II.2.2.3.2. Détermination des différents coefficients de pondération (Cp) et vérification de la cohérencelogique \\ Le coefficient de pondération de chaque facteur correspond à} l'intensité de son impact dans l'étude de la vulnérabilité à l'inondation. Leur détermination se fait en deux étapes :

Etape 1: Déterminer le Vecteur propre $(\mathrm{Vp})$ de chaque facteur

$$
V p=\sqrt[k]{w 1 x \ldots \ldots \ldots x w k}
$$

Avec W1, W2,....WK, les différentes valeurs attribuées aux différents facteurs lors de leur comparaison et $\mathbf{k}$ le nombre de paramètres comparés. 
Etape 2: Calculer le Coefficient de pondération $(\mathrm{Cp})$ de chaque facteur :

$$
C p=\frac{V p}{\sum \quad V p}
$$

Avec Vp le Vecteur Propre du facteur dont on veut calculer le Cp et $\Sigma \mathrm{Vp}$, la somme de tous les vecteurs propres calculés.

La somme des $\mathrm{Cp}$ de tous les paramètres d'une matrice doit être égale à $\mathbf{1}$.

Avant la détermination des différents coefficients de pondération, la cohérence des jugements doit être vérifiée afin de valider les priorités obtenues. Etant donné que cette méthode repose sur la subjectivité, les situations de cohérence parfaite sont rares.

En effet, La cohérence logique est un ratio qui permet de vérifier ou de valider la cohérence de la matrice originelle. Ce ratio peut être interprété comme la probabilité que la matrice soit complétée d'une manière aléatoire.

Dans le cas où la valeur du Ratio de Cohérence dépasse $10 \%$, les appréciations peuvent exiger certaines révisions (Saaty,1980). Ainsi, le Ratio de Cohérence est donné par l'équation suivante :

$$
R C=\frac{I C}{I A}
$$

Avec $:$ IC = Indice de Cohérence et IA = Indice Aléatoire

Les valeurs de IA sont données en fonction du nombre de facteurs comparés et ces valeurs ont été déjà déterminées par Saaty. Le tableau IV, présente ces différentes valeurs.

Tableau IV: Indices aléatoires en fonction du nombre d'éléments comparés (Saaty, 1977)

\begin{tabular}{lllllllllll}
\hline $\begin{array}{l}\text { Nombre de } \\
\text { critères }\end{array}$ & 2 & 3 & 4 & $\mathbf{5}$ & 6 & 7 & 8 & 9 & 10 & 11 \\
\hline IA & 0 & 0,58 & 0,90 & $\mathbf{1 , 1 2}$ & 1,24 & 1,32 & 1,41 & 1,45 & 1,49 & 1,51
\end{tabular}

Dans le cadre de cette étude, IA $=1,12$ correspondant à 5 facteurs comparés.

Connaissant IA, la détermination de IC qui selon la méthode AHP indique la probabilité que les estimations ont été aléatoirement assignées suit les étapes suivantes :

- étape $1:$ normaliser la matrice originale en divisant chaque élément d'une colonne par la somme de cette colonne ;

- étape 2 : faire la moyenne de chaque ligne pour déterminer le vecteur prioritaire $[\mathrm{C}]$;

- étape 3: multiplier chaque colonne de la matrice par le vecteur prioritaire lui correspondant pour déterminer la priorité globale [D] ; 
- étape 4 : diviser chaque priorité globale par le vecteur prioritaire lui correspondant afin de déterminer la priorité rationnelle $[\mathrm{E}]$;

- étape 5 : déterminer la moyenne des priorités rationnelles $(\lambda \max )$;

- $\lambda \max =\frac{[E]}{k}$

- étape $6:$ calculer l'Indice de Cohérence (IC) $:$ IC $=\frac{\lambda \max -k}{k-1}$

On déduit enfin le Ratio de Cohérence qui est égale $: \mathrm{RC}=\frac{I C}{I a}$

Selon les calculs effectués, le RC $<10 \%$ (car Rc $=4 \%$ ). Par conséquent, le raisonnement utilisé pour la matrice est cohérent.

Tous les résultats obtenus sont consignés dans le tableaux V:

Tableau V: Résultats des différents calculs effectués

\begin{tabular}{|c|c|c|c|c|c|c|c|c|c|c|c|c|c|}
\hline & OS & $\mathbf{P}$ & DD & $\mathbf{F E}$ & DP & Cp & ' & Elig & $\mathbf{E}$ & C & $\lambda \max$ & IC & RC \\
\hline OS & 0,56 & 0,65 & 0,52 & 0,43 & 0,36 & 0,51 & & 2,52 & 5,26 & 0,54 & \multirow{6}{*}{5,19} & \multirow{6}{*}{0,05} & \multirow{6}{*}{$\begin{array}{l}0,0 \\
4\end{array}$} \\
\hline$P$ & 0,19 & 0,21 & 0,32 & 0,31 & 0,28 & 0,26 & & 1,31 & 5,18 & 0,28 & & & \\
\hline DD & 0,11 & 0,07 & 0,10 & 0,18 & 0,2 & 0,13 & & 0,66 & 5,19 & 0,13 & & & \\
\hline FE & 0,08 & 0,04 & 0,04 & 0,06 & 0,12 & 0,06 & & 0,34 & 5,13 & 0,06 & & & \\
\hline DP & 0,06 & 0,03 & 0,02 & 0,02 & 0,04 & 0,03 & & 0,17 & 5,20 & 0,034 & & & \\
\hline$\Sigma$ col & 1,00 & 1,00 & 1,00 & 1,00 & 1,00 & 1,00 & & 5,00 & 25,96 & 1 & & & \\
\hline
\end{tabular}

\section{II.2.2.3.3 Agrégation des facteurs}

Elle consiste en la sommation des valeurs standardisées et pondérées de chaque facteur ou critère intervenant dans l'élaboration de l'indicateur donné. On peut résumer cette approche par la formule suivante :

$$
S=\sum_{i=2}^{n} W i X i
$$

$S$ : résultat;

Wi: poids du facteur i ;

Xi: valeur standardisée du facteur i

La carte de vulnérabilité est ainsi obtenue à partir de la formule suivante

Carte de Vulnérabilité $=0,51 \times(O S)+0,26 \times(P)+0,13 \times(D D)+$ $0,06 \times(F A)+0,03 \times(D P)$

Pour chaque paramètre, le poids $(\mathrm{Cp})$ est multiplié par les différentes cotes correspondantes. Ce calcul est effectué avec l'outil " Raster Calculator " 
du sous module " Map Algebra " du logiciel ArcGIS. Ce calcul permet d'obtenir des intervalles d'indices auxquels des classes de vulnérabilité sont attribuées.

\section{Resultats et Discussion}

\section{II1.1 Carte de vulnérabilité à l'inondation de la commune de Bingerville} La combinaison des cinq critères reclassifiés a permis d'obtenir la carte de vulnérabilité à l'inondation de la commune de Bingerville ( Figure7).

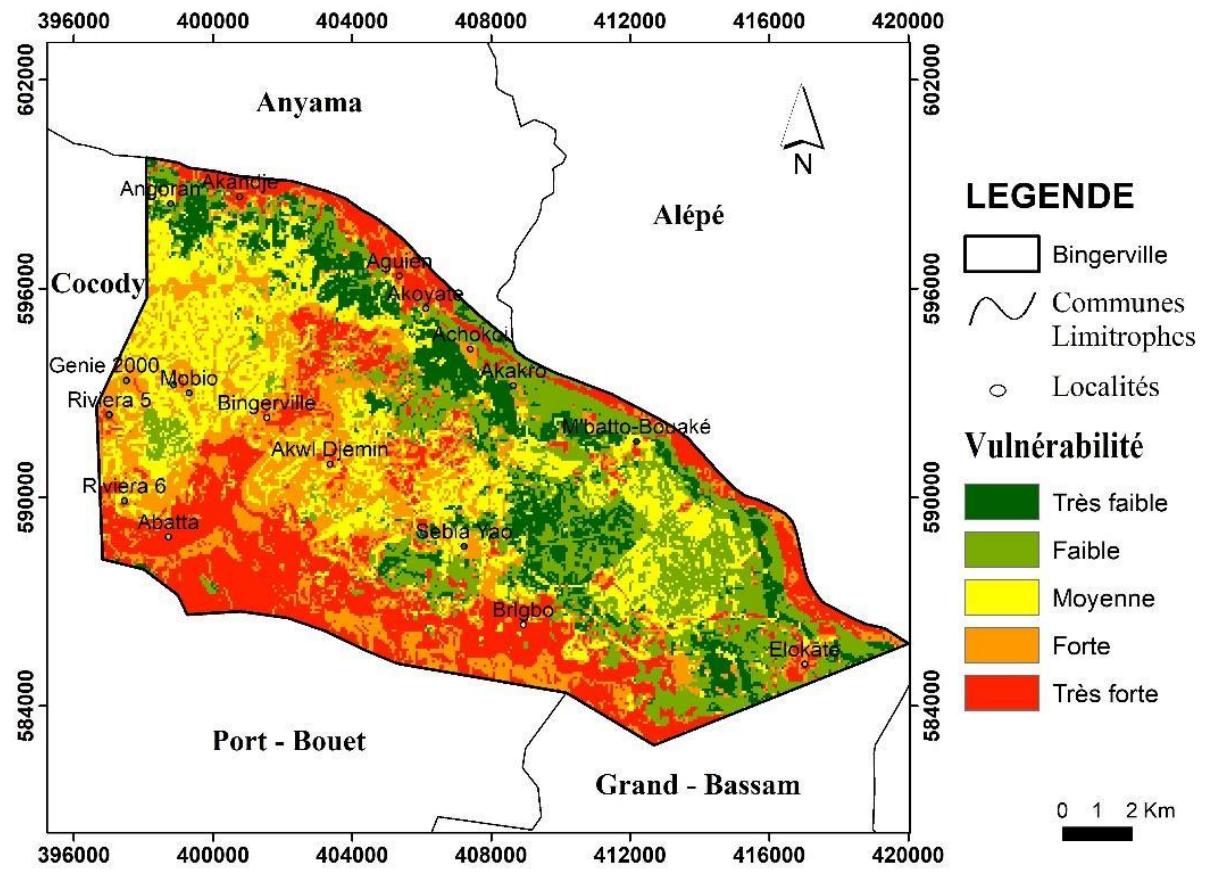

Figure 7: Carte de vulnérabilité à l'inondation de la commune de Bingerville

Cette carte présente cinq classes de vulnérabilité allant de très faible à très forte. L'analyse par classe permet de dire que, les classes de vulnérabilité très faible et faibles sont rencontrées dans certains endroits notamment au Nord et à l'Est et partiellement au Sud-Ouest de la commune. Elles représentent respectivement $11,59 \%$ et $20,70 \%$ de la superficie de la commune. Elles correspondent aux zones de pentes fortes, de drainage moyen et de forêt prédominante.

La classe de vulnérabilité moyenne occupe environ $21,88 \%$ de la superficie de la zone d'étude et est rencontrée dans les parties Ouest, Centre et partiellement à l'Est de la commune. Ce sont des zones de sols nus, ou avec une prédominance de bâtis et ayant des drainages moyens et forts sur des pentes moyennes. Il s'agit en majorité du centre-ville de la commune et de la partie partagée avec la commune de Cocody. 
Les classes de vulnérabilité forte et très forte représentent respectivement $22,55 \%$ et $23,26 \%$ de la superficie totale de la zone d'étude. Elles se situent en majeure partie à l'Ouest de la commune, précisément au niveau du centre-ville de Bingerville, et dans certaines localités bordées par les lagunes. Ces classes correspondent aux zones de faibles à moyennes pentes, de faible densité de drainage, de forte densité des facteurs anthropiques et de population dont la prédominance est composée de bâtis et de sol nu.

En somme, en tenant compte de la répartition des surfaces habitées (le centre-ville, quelques villages et les quartiers partagés avec la commune de Cocody), la commune de Bingerville peut être considérée comme ayant une prédominance de vulnérabilité moyenne à l'inondation.

\section{III.2. Validation de la carte de vulnérabilité à l'inondation}

La vulnérabilité à l'inondation de la commune de Bingerville est liée en partie à la perméabilité de son sol et surtout à sa capacité à drainer l'eau. La carte de répartition des perméabilités de la nappe de Bonoua (Hydroexpert, 2000) montre que le sol de Bingerville présente une perméabilité faible dont les valeurs sont comprises entre $2.10^{-4}$ et $3.10^{-4} \mathrm{~m} / \mathrm{s}$, ce qui explique son taux de ruissellement élevé par rapport à celui de l'infiltration (Figure 8).

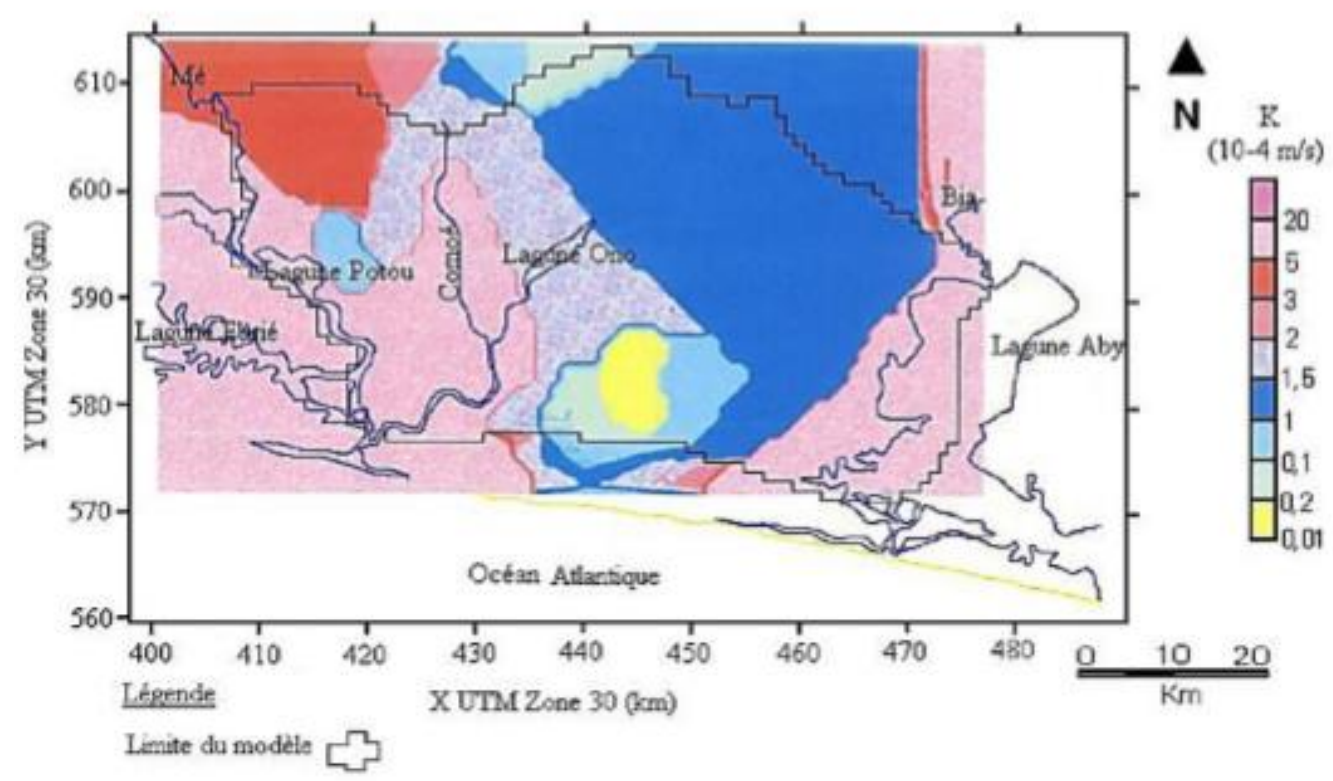

Figure 8: Carte de répartition des perméabilités de la nappe de Bonoua (Hydroexpert, 2000)

L'on remarque que les zones de vulnérabilité forte observées sur la carte de vulnérabilité coïncident avec les zones présentant une densité élevée des facteurs environnementaux (cf.Tableau II). Cette forte vulnérabilité est 
aussi observée dans les endroits occupés par les lagunes et dans les localités situées à proximité de celles-ci (Abatta, Bregbo, Eloka Té, Achokoi, Aghien et Akandjé).

\section{III.3. Facteurs anthropiques}

Les résultats des investigations socio-environnementales dans la commune indiquent que deux villages (Angorankoi et Abatta) et sept quartiers (Harris, Sicogi 2, Blanchon, Berlin, Gbagba, Korokobougou et EECI) sont potentiellement confrontés aux inondations liées aux facteurs environnementaux. Il s'agit des dépôts sauvages d'ordures sur les voies ou dans les réseaux d'évacuation d'eau avec souvant leur destruction volontaire ou involontaire (réseau dysfonctionnel), les constructions dans les bassins d'orage, ou sur les talwegs de drainnage (Figure 9).

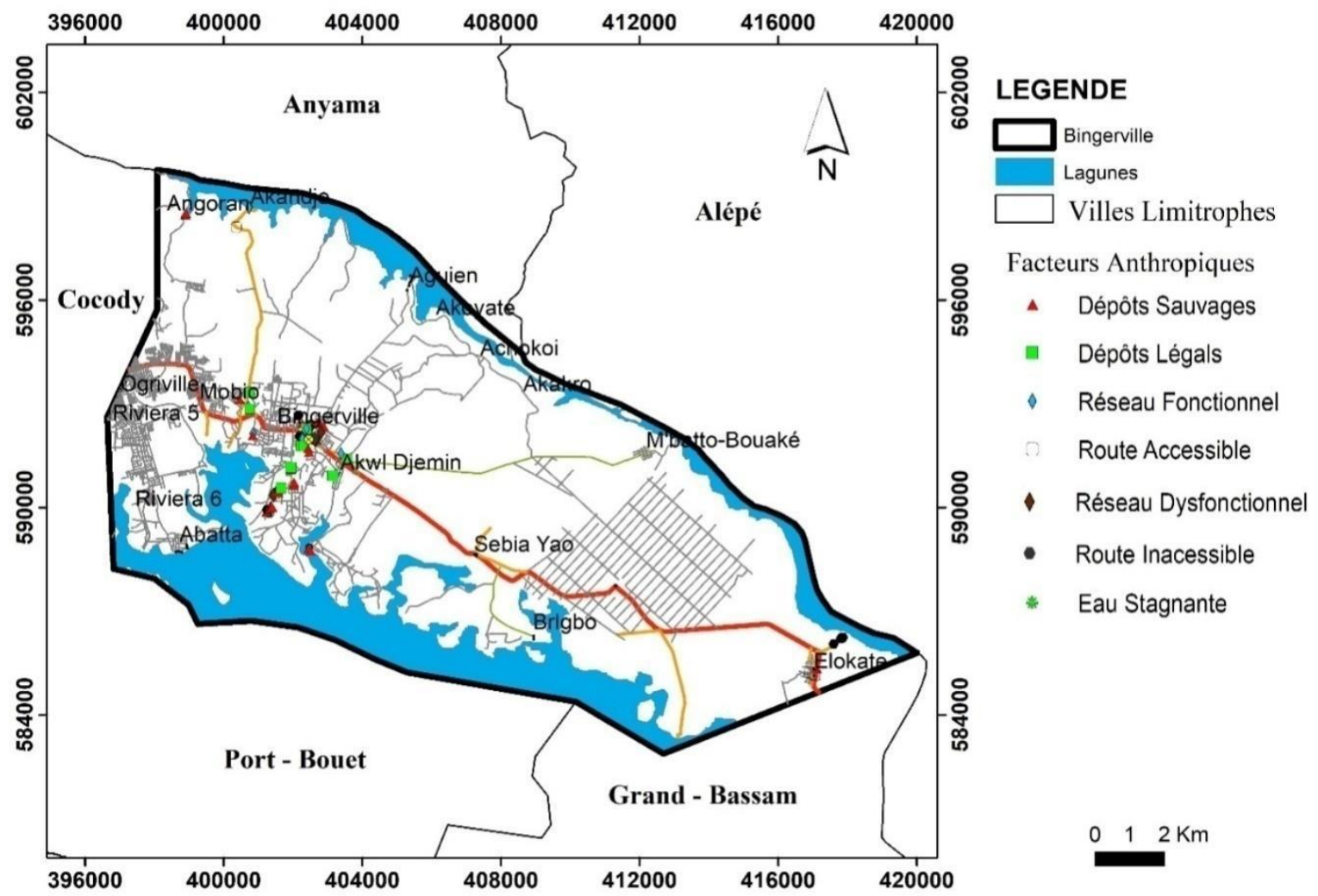

Figure 9 : Distribution spatiale des facteurs environnementaux anthropiques

La figure 9 présente quelques uns de ces facteurs environnementaux anthropiques relevés. Ils sont plus nombreux au niveau du centre-ville (Bingerville, Sicogi 2, etc.) par rapport aux quartiers situés à sa périphérie. Ces facteurs environnementaux qui traduisent le comportement des populations 
dans cette commune sont de nature à empêcher la circulation effective des eaux des lits naturels et artificiels occasionnant ainsi des inondations temporelles ou permanantes.

\section{III.4. Discussion}

Dans cette étude, l'utilisation de la méthode d'analyse multicritères de Saaty pour la cartographie des zones vulnérables et potentiellement inondables de la commune de Bingerville permet une compréhension plus exhaustive du phénomène étudié. Cependant, elle rencontre des difficultés au niveau de la pondération inter-critère des différents critères choisis et les notes assignées aux différentes classes de ces critères. En effet, la subjectivité des poids et des cotes assignés aux critères dans l'application de l'analyse multicritère est un facteur limitant (Eba et al., 2013). Cette étape peut donc parfois être rendue difficile quand les connaissances sont réduites, et avec la subjectivité que cela comporte. Ensuite, l'analyse multicritère utilisée fait un certain nombre de simplifications selon la logique mathématique. Elle applique implicitement l'hypothèse que tous les critères considérés dans la réalisation d'une carte thématique, sont parfaitement comparables.

Bien que présentant certains challenges, 1 'analyse multicritère a permis d'assurer la parfaite synthèse des différents facteurs utilisés à savoir : les pentes, la densité de drainage, l'occupation du sol la densité de population et les paramètres environnementaux anthropiques. Cette synthèse a conduit à la production d'informations utiles pour une bonne gestion du phénomène d'inondation. Cette méthode d'analyse multicritère a été utilisée par plusieurs auteurs dans des travaux antérieurs portant sur la vulnérabilité de plusieurs phénomènes environnementaux dont la pollution des ressources en eau, (Jourda et al., (2007), Kouamé (2007), Eba et al., (2013)), la vulnérabilité des terres au risque d'érosion, (Balliet, 2017) et le risque d'inondation, (Danumah (2013), Sorokoby et al., (2013), Balliet (2017), Yéo (2017)).

En outre, les résultats obtenus dans cette étude ont montré que la commune de Bingerville est moyennement vulnérable à l'inondation, dans son ensemble, mais avec, des poches de fortes vulnérabilités observées dans certains quartiers situés principalement au centre-ville, en zones périphériques frontière avec la commune de Cocody et certains villages situés en bordure de lagune tels que Anan, Akandjé et Abatta. Ces résultats concordent avec ceux de Balliet (2017) dans la région de Gôh et de Yéo (2017) dans la commune de Cocody. Les travaux de ces auteurs ont révélé une vulnérabilité moyenne due à la mise à nu des terres, réalisée dans la région de Gôh et par Yéo (2017) dans la commune de Cocody. Ces résultats sont aussi en accord avec ceux obtenus dans les travaux antérieurs de Saley et al., (2005), Abdellah (2008), Aroua et Berezowska-Azzag (2009). Selon ces auteurs, le risque à l'inondation est lié à l'action combinée de nombreux facteurs parmi lesquels l'urbanisation non 
contrôlée, l'activité anthropique, l'intensité des pluies, les pentes faibles et l'occupation des sols.

Les résultats des enquêtes socio-environnementales montrent que les zones les plus touchées par les inondations dans la commune de Bingerville sont les quartiers tels que Sicogi 2, Blanchon, EECI, Korokobougou.

Ces zones sont régulièrement affectées lors d'épisodes pluvieux. Cela est dû à leurs situations géographiques et aussi à l'absence de système d'assainissement adéquat. Les villages en bordure de lagunes (Anan, Akandjé, Abatta) quant à eux sont confrontés aux mouvements d'avancée de la lagune ce qui affecte seulement les habitations situées en bordure.

En effet, les résultats de ce travail sont fiables et peuvent être utilisés comme système d'alerte à l'endroit des autorités de la commune. Ils peuvent également guider leurs interventions dans leur prise de décision pour une gestion intégrée et durable des crises d'inondation et de la prévention du phénomène.

\section{Conclusion}

La spatialisation des facteurs environnementaux naturels et anthropiques a permis de mieux mettre en évidence et comprendre leurs implications dans le phénomène d'inondation, dans la commune de Bingerville. Cette étude a été possible grâce aux outils de cartographie et de SIG. L'application de la méthode d'analyse multicritère a permis de combiner les cartes d'occupation du sol, de pentes, de densité de drainage, de densité de populations et de densité des facteurs environnementaux anthropiques afin d'obtenir la carte de vulnérabilité à l'inondation de la commune de Bingerville. Cette carte comporte cinq classes de vulnérabilité à savoir : très faible ; faible ; moyenne; forte ; et très forte.

Au terme de cette étude, il ressort que la commune de Bingerville est moyennement vulnérable à l'inondation dans son ensemble, à cause de son relief qui présente des pentes à certains endroits et l'occupation rationnelle des terrains, facilitant le drainage des eaux pluviales. Cependant, les constructions anarchiques, les ouvrages routiers et les ouvrages de drainage en mauvais état ne permettent pas le bon drainage des eaux à certains endroits (quartiers Blanchon et Sicogi 2).

La carte obtenue constitue un outil d'aide à la décision qui peut ainsi aider les gestionnaires de la commune, afin de prendre d'ordre et déjà des dispositions pour éviter dans un futur proche des catastrophes liées aux inondations telles que connues dans la commune voisine, c'est-à-dire Cocody. 


\section{References :}

1. Abdellah B. (2008). Risques d'inondation et occupation des sols dans le thore (region de labruguiere et de mazamet), mémoire présenté pour l'obtention de master I de géographie et aménagement, Institut Daniel Faucher, 75p.

2. Aké G. E., Eba A. E. L., Assi J. J., Kouadio B. H., Biemi J.(2019). Apport des SIG à la délimitation des périmètres de protection autour de la prise d'eau de la SODECI à Aboisso, Sud-Est de la Côte d'Ivoire. Afrique Sciences 15 (3) (2019) 234-252, ISSN 1813-548X.

3. Arouna N., Berezowska-Azzag E. (2009). Contribution à l'étude de la vulnérabilité urbaine au risque d'inondation dans un contexte de changement climatique. Cas de la vallée de Oued El Harrach à Alger, Symposium, $20 \mathrm{p}$.

4. AssaléP., Monde S., Aka K. (2012). Caractérisation lithostratigraphique et petro-sedimentologique des formations tertiaire-quaternaires de la région de Bingerville (Côte d'Ivoire), Afrique Science: Revue internationale des Sciences et Techniques 8(2), pp93-112.

5. Balliet René (2017). «Etude des impacts du changement climatique et de la pression anthropique sur l'environnement de la région du gôh (centre-ouest de la côte d'ivoire): analyse de mesures d'adaptation endogène » Thèse de doctorat, Université Félix Houphouët Boigny, Abidjan. 192

6. Chérif S. (2014). Risque climatique et réactivité des populations urbaines vulnérabilisées face à la montée des eaux de mer de Gonzagueville, Abidjan (Côte d'ivoire); (VertigO) la revue électronique en science de l'environnement, Vol $14 \mathrm{~N}^{\circ} 1,2014,16 \mathrm{p}$.

7. Danumah J. H. (2013). Cartographie des zones à risques d'inondations. Apport des Systèmes d'Informations Géographiques (SIG) et de l'imagerie satellitaire. Application au bassin versant de la Davo (SASSANDRA). Mémoire de Master, Université Félix Hophouët Boigny, Abidjan. 77 p.

8. Douay D. et Lardieg E. (2010).Délimitation des Aires d'Alimentation des captages prioritaires du bassin Adour Garonne, Méthodologie de cartographie de la vulnérabilité intrinsèque des captages d'eau superficielle, 28p.

9. Eba A. E. L., Kouame K. J., Jourda J.P., Saley M. B., Ake G. E., Deh Serge K. and Anoh K. A. (2013). Demarcation of Surface Water Protection Perimeters by Using GIS: Case of Gagnoa Reservoir in West Central of Côte d'Ivoire. International Journal of Scientific \& Engineering Research, Volume 4, Issue 4, pp.1311-1320. 
10. El Morjani Z. (2002). Conception d'un système d'information à référence spatiale pour la gestion environnementale ; application à la sélection de sites potentiels de stockage de déchets ménagers et industriels en région semi-aride (Souss, Maroc). Thèse de Doctorat, Université de Genève, Terre et Environnement Vol. 42, 300 p.

11. Haouchine A., Boudoukha A., Fatima Z. H. Et Rachid N. (2010). Cartographie de la recharge potentielle des aquifères en zone aride. Eurojournals, 201045 (4), pp 1-13.

12. Hydroexpert (2000). Réalisation d'un modèle hydrodynamique de la nappe Sud-comoé, $51 \mathrm{p}$.

13. 13.INS. (2014). Institut National de la Statistique: Recensement Général de la Population et de l'Habitat, 26 p.

14. Jeune Afrique- Economique (17 Aout 2020). Ville et Climat: Abidjan traque les pluies diluviennes. Dossier article de presse $<<$ Comment les villes africaines font face au risque climatique>>.

15. Jourda J. P., Kouame K. J., Adja M. G., Deh S. K., Anani A. T, Efini A. T. et Biemi J. (2007). Evaluation du degré de protection des eaux souterraines : vulnérabilité à la pollution de la nappe de Bonoua (SudEst de la Côte d'Ivoire) par la méthode DRASTIC. Acte de conférence Francophone ESRI 10 et 11 octobre-Versailles. 18 p.

16. Kablan M.K.A. (2017). Vulnérabilité et adaptation des populations urbaines aux effets des variations climatiques (température et pluviométrie): analyse de la situation dans la commune de Cocody, Abidjan, côte d'ivoire. Thèse de doctorat, Université Félix Houphouët Boigny, Abidjan. 242 p.

17. Koffi A. B. (2016). Détermination des zones de recharges de la nappe de Bonoua : Approche cartographique et SIG, Mémoire en Science de la Terre, Univ. Abidjan. 70p.

18. Koffi Y.B., Ahoussi K.E., Kouassi A.M., Biémi J. (2014). Ressources minières, pétrolières et gazières de la côte d'Ivoire et problématiques de la pollution des ressources en eau et des inondations, Geo-Eco-Trop. (2014),38, 1, n.s. : pp119-136.

19. Kouamé K.J.(2007). Contribution à la Gestion Intégrée des Ressources en Eaux (GIRE) du District d'Abidjan (Sud de la Côte d'Ivoire) : Outils d'aide à la décision pour la prévention et la protection des eaux souterraines contre la pollution. Thèse de doctorat, Université de Cocody, $229 \mathrm{p}$.

20. Mulder M. A. (2001). Advance in the application of remote sensing and GIS for surveying mountainous land. International Journal of Applied Earth Observation and Geoinformation, vol. 3 no 1,p. 3-10.

21. Musy A. Soutter M. (1991). Physique du sol. Presse Polytechniques et Universitaires Romandes, Lausanne, Suisse, 335p. 
22. Nouaceur Z. (2015). les capitales sahéliennes face à la recrudescence des inondations urbaines, Territorium $\mathrm{N}^{\circ} 22$ (131-140) ISSN : 0872 8941, 2015, 132p.

23. Saaty T. L. (1977). A scaling method for priorities in hierachical structures. J. Math. Psychology, 15, pp. 234-281.

24. SaatyT. L. (1980). The Analytic Hierarchy Process: Planning, Priority setting, Resource allocation, McGraw-Hill, New York, 19 p.

25. Saley M.B., Kouamé K.F., Penven M.J., Biémi J. et Kouadio B.H. (2005). Cartographie des zones à risque d'inondation dans la région semi-montagneuse à l'Ouest de la Côte d'Ivoire : Apports des MNA et de l'imagerie satellitaire. Télédétection, vol. 5, n ${ }^{\circ}$ 1-2-3, pp. 53-67.

26. Sorokoby V. M. (2013). Étude hydrologique et hydrogéologique de la région de Soubré (Sud-ouest de la Côte d'Ivoire) dans un contexte de variabilité climatique. Thèse Unique. Université Félix Hоирhоuёt Boigny. Abidjan (Côte d'Ivoire), $171 \mathrm{p}$

27. Tah N. J., Dje K. B., Koli Bi Z., Kangah D. P., Coulibaly K. A., Kouadio Z. A. (2018). Caractérisation du ruissellement dans le district d'Abidjan, (Journal de la Recherche Scientifique de l'Université de Lomé) Journal Home, Vol $20 \mathrm{~N}^{\mathrm{O}} 2$.

28. 27.Yeo D. (2017). Phénomène d'inondation à Abidjan : Analyse de quelques traits caractéristiques dans la commune de Cocody, Mémoire en Sciences de la Terre, Univ. Abidjan, 64p. 